\title{
Nonclassical Properties of Even and Odd Semi-Coherent States
}

\author{
H. H. Salah \\ Department of Physics, Faculty of Science (Girls Branch), Al-Azher University, Nasr City, Cairo, Egypt
}

\begin{abstract}
Even and odd semi-coherent states have been introduced. Some of the nonclasscial properties of the states are studied in terms of the quadrature squeezing as well as sub-Poissonian photon statistics. The HusimiKano Q-function and the phase distribution in the framework of Pegg and Barnett formalism, are also discussed.
\end{abstract}

Keywords: Semi-coherent states, quadrature squeezing, sub-Poissonian statistics, quasi-probability distribution function ( $Q$ function), Phase distribution.

\section{Introduction}

Over the past four decades, various works were devoted to the study of coherent states (CSs) and their application in different branches of physics from both theoretical and experimental points of view. The concept of CSs was first introduced by Schrodinger as Gaussian wave-function that could be constructed from a particular superposition of the wave functions corresponding to the discrete eigenvalues of the harmonic oscillator [1]. These states prove a close connection between quantum and classical formulations of a given physical system. The importance of CSs was put forward by Glauber $[2,3]$ in quantum optics as eigenstates of the annihilation operator $\hat{a}|z\rangle=z|z\rangle$, which he realized that these states have interesting property of minimizing the Heisenberg uncertainty relation, since the product of their uncertainties in the position and momentum saturates the Hiesenberg uncertainty relation with equal values in each quadrature. Moreover CSs were reintroduced by Klauder in $[4,5]$ by action of the Glauber displacement operator, $D(z)=e^{z \hat{a}^{+}-\bar{z} \hat{a}}$, on the vacuum states, where $\mathrm{z}$ is a complex variable. These states can be served as a starting point to introduce nonclassical states that have attracted considerable attention in quantum optics over the last decades. Over the last four decades there have been several experimental demonstrations of non-classical effects, such as photon antibunching [6], sub-Poissonian statistics [7] and squeezing [8]. Also, significant progress was achieved in the development of practical realizable schemes of nonlinear quantum states, such as f-CS or nonlinear CS [9-15], even and odd CS $[8,16]$ and semi CS [17]. These states have attracted much attention in recent decades due to their helpful applications in various fields of research [10, 18-22].

For instance, the concept of the semi-CSs was introduced in [23] as the states of a harmonic oscillator which possess time-independent values of the quadrature variances $\sigma_{x}$ and $\sigma_{p}$, different from the vacuum (or coherent state) values. These states are different from the CSs in not having the minimum value $\frac{1}{2} \hbar$ for the uncertainty product $\Delta x . \Delta p$. They have shown that the necessary and sufficient condition for such states is

$$
\left\langle\hat{a}^{2}\right\rangle=\langle\hat{a}\rangle^{2},
$$

Where $\hat{a}=(\hat{x}+i \hat{p}) / \sqrt{2}$ is the usual bosonic annihilation operator (using the units $\hbar=m=\omega=1$ ). Condition (1) is obviously satisfied for the usual coherent states $|\alpha\rangle$, for which $\hat{a}|\alpha\rangle=\alpha|\alpha\rangle$. Another trivial example is the Fock state $|n\rangle$, for which $\left\langle n\left|\hat{a}^{2}\right| n\right\rangle=0$.

An immediate consequence of equation (1) is the equality of the quadrature variances, $\sigma_{x}=\sigma_{p}$, and zero value of the covariance, $\frac{1}{2}\langle\hat{x} \hat{p}+\hat{p} \hat{x}\rangle-\langle\hat{x}\rangle\langle\hat{p}\rangle=0$. Thus 'semi-coherent' states cannot exhibit usual (second-order) squeezing.

A nontrivial example of 'semi-coherent' states, only briefly discussed in [23], is a normalized superposition of two coherent states of the form

$$
\left|\alpha_{\perp} \beta\right\rangle=\frac{|\alpha\rangle-|\beta\rangle\langle\beta \mid \alpha\rangle}{\left(1-|\langle\beta \mid \alpha\rangle|^{2}\right)^{1 / 2}} .
$$


The notation $\left|\alpha_{\perp} \beta\right\rangle$ means that the state (2) is orthogonal to the state $|\beta\rangle$ :

$$
\left\langle\beta \mid \alpha_{\perp} \beta\right\rangle=0 \text {. }
$$

Therefore, the state $\left|\alpha_{\perp} \beta\right\rangle$ can be considered as an orthogonal projection of the coherent state $|\alpha\rangle$ to another coherent state $|\beta\rangle$. In the state (2) one has [23]

$$
\sigma_{x}=\sigma_{p}=\frac{1}{2}+\frac{|\langle\beta \mid \alpha\rangle|^{2}|\alpha-\beta|^{2}}{1-|\langle\beta \mid \alpha\rangle|^{2}}
$$

so that, indeed, $\sigma_{x}=\sigma_{p}>\frac{1}{2}$.

Later Another investigation have demonstrated that firstly, state (2) has the same average value of the annihilation operator $\hat{a}$ as the coherent state $|\alpha\rangle,\left\langle\alpha_{\perp} \beta|\hat{a}| \alpha_{\perp} \beta\right\rangle=\alpha$.for any value of $\beta$, secondly, a possibility of introducing a large family of 'generalized semi-coherent states', which satisfy property $\left\langle\hat{a}^{n}\right\rangle=\langle\hat{a}\rangle^{n}=\alpha^{n}$ for an arbitrary operator $\hat{A}$ ( All such states have the same structure as the superposition (2), where the components $|\alpha\rangle$ and $|\beta\rangle$ should be understood as eigenstates of the operator $\hat{A}$ and thirdly semiCSs possess other nonclassical properties, in particular, the possibility of the fourth-order squeezing and subPoissonian statistics [17]. Recently, it has been demonstrated that Semi-CSs as superposition of two coherent states, are suasible states for considering upper bounds for the relative energy difference with a fixed fidelity between them [24]. Also a new family of semi-CSs for a charged particle moving in a constant magnetic field have been introduced [25]. These states interpreted as a nonlinear coherent states (NLCSs) with a special nonlinearity function. Another investigation demonstrated that, the semi-CSs can be considered as a special case of nonlinear semi-CSs when $\mathrm{f}(\mathrm{n})=1$. Then, the nonclassical properties such as quadrature squeezing, amplitude-squared squeezing, fourth-order squeezing, number and phase squeezing and sub-Poissonian statistics for the introduced states in addition to their Pegg-Barnett phase distribution are generally studied [26].

The main goal of this paper is the introducing of even and odd semi-CSs. Our motivation is to search for more nonclassical properties in the obtained states, since, as reported in [17, 23], the semi-CSs only possess sub-Poissonian statistics and fourth-order squeezing. The paper is organized as follows. In section II, we introduce the even and odd semi-CSs. Certain nonclassical properties associated with these states are deduced, in section III, such as the quadrature squeezing, sub-Poissonian distribution, the Husimi-Kano Qfunction and Phase distribution. Finally the conclusion is presented in section IV.

\section{Introducing Even and Odd Semi-CSs (ESemi-CSs and OSemi-CSs)}

The discrete energy values corresponding to motion of a charged particle on the infinite flat surface in the presence of a uniform external magnetic field perpendicular to this plane are called Landau levels [27]. The exact solvability of the symmetric-gauge Landau Hamiltonian,

$$
H=\hbar \omega\left(a^{+} a+\frac{1}{2}\right)=\hbar \omega\left(b^{+} b+\frac{1}{2}\right)-\omega L_{3} \text { with } L_{3}=-i \hbar \partial / \partial \varphi
$$

have been considered. It includes an infinite-fold degeneracy on the Landau levels [28], i.e.

$$
H|n, m\rangle=\hbar \omega\left(n+\frac{1}{2}\right)|n, m\rangle,
$$

Where Landau cyclotron frequency, $\omega$, is expressed in terms of the value of the electron charge, its mass, the magnetic field strength $\mathrm{B}$ and also the velocity of light as $\omega=e B / M c$. The number $\mathrm{m}$ is an integer and $\mathrm{n}$ is a non-negative one together with $n \geq-m$ limitation. Each pair of creation and annihilation operators of the system, $\left(a, a^{+}\right)$and $\left(b, b^{+}\right)$, form two separate copies of Weyl-Heisenberg algebra,

$$
\left[a, a^{+}\right]=1,\left[b, b^{+}\right]=1,\left[a, b^{+}\right]=\left[a^{+}, b\right]=[a, b]=\left[a^{+}, b^{+}\right]=0,
$$

With the unitary representations as

$$
\begin{aligned}
a|n, m\rangle & =\sqrt{n}|n-1, m+1\rangle \quad, \quad & a^{+}|n-1, m+1\rangle=\sqrt{n}|n, m\rangle, \\
b|n, m\rangle & =\sqrt{n+m}|n, m-1\rangle, \quad & b^{+}|n, m-1\rangle=\sqrt{n+m}|n, m\rangle .
\end{aligned}
$$

The creation and annihilation operators of the system are used to express the coordinates $\mathrm{x}$ and $\mathrm{y}$ and their conjugate momenta, i.e., $p_{x}$ and $p_{y}$, 


$$
\begin{aligned}
& x=\sqrt{\frac{\hbar}{2 M \omega}}\left(b+b^{+}-a-a^{+}\right), \quad p_{x}=\frac{i}{2} \sqrt{\frac{M \hbar \omega}{2}}\left(b^{+}-b+a-a^{+}\right), \\
& y=i \sqrt{\frac{\hbar}{2 M \omega}}\left(b+b^{+}-a-a^{+}\right), \quad p_{y}=\frac{1}{2} \sqrt{\frac{M \hbar \omega}{2}}\left(b^{+}+b+a+a^{+}\right),
\end{aligned}
$$

Using the constructed the minimum uncertainty two variable CSs, $|\beta, \alpha\rangle$ for a charged particle in the magnetic field as [28],

$$
|\beta, \alpha\rangle=e^{\beta a^{+}-\bar{\beta} a}|\alpha\rangle_{0}^{b}=e^{-|\beta|^{2} / 2} \sum_{n=0}^{\infty} \frac{\beta^{n}}{\sqrt{n !}}|\alpha\rangle_{n}^{b}
$$

where they satisfy following eigenvalue equations

$$
\begin{aligned}
& a|\beta, \alpha\rangle=\beta|\beta, \alpha\rangle, \\
& b|\beta, \alpha\rangle=\alpha|\beta, \alpha\rangle .
\end{aligned}
$$

Also, $|\alpha\rangle_{n}^{b}$ are the normalized Glauber CSs, which are obtained by using the displacement operator $D(\alpha)=e^{\alpha b^{+}-\bar{\alpha} b}$ acting on the Landau level $|n,-n\rangle$ :

$$
|\alpha\rangle_{n}^{b}=D(\alpha)|n,-n\rangle=e^{-\frac{|\alpha|^{2}}{2}} \sum_{m=-n}^{\infty} \frac{\alpha^{n+m}}{\sqrt{(n+m) !}}|n, m\rangle
$$

By using the orthonormall relation of Landau levels, i.e., $\left\langle n^{\prime}, m^{\prime} \mid n, m\right\rangle=\delta_{n^{\prime} n} \delta_{m^{\prime} m}$, one can deduce that coherent states $|\alpha\rangle_{n}^{b}$ and $|\alpha\rangle_{m}^{a}$ form two separate complete bases, that is, ${ }_{n}^{b}\langle\alpha \mid \alpha\rangle_{n^{\prime}}^{b}=\delta_{n^{\prime} n}$ and ${ }_{m}^{a}\langle\alpha \mid \alpha\rangle_{m^{\prime}}^{a}=\delta_{m^{\prime} m}$. As mentioned in previous section, $|\beta, \alpha\rangle$ satisfy the condition (1) and thus can be regarded as semi-CSs, which can be introduced by substituting state $|\alpha\rangle_{n}^{b}$ (12) into (10) the state $\left|\beta_{\perp} \alpha\right\rangle$ can be rewritten in the Fock basis as

$$
\left|\alpha_{\perp} \beta\right\rangle=\frac{1}{N} \sum_{n=0}^{\infty} \frac{e^{-\frac{|\alpha|^{2}}{2}} \alpha^{n}-\langle\beta \mid \alpha\rangle e^{-\frac{|\beta|^{2}}{2}} \beta^{n}}{\sqrt{n !}}|n\rangle .
$$

2 (a) ESemi-CSs:

From equation (13) ESemi-CSs is given as

$$
\left|\alpha_{\perp} \beta\right\rangle_{e}=\frac{1}{N_{e}} \sum_{n=0}^{\infty} C_{2 n}|2 n\rangle
$$

Where,

$$
C_{2 n}=\frac{1}{N_{e}} \frac{e^{-\frac{|\alpha|^{2}}{2}} \alpha^{2 n}-\langle\beta \mid \alpha\rangle e^{-\frac{|\beta|^{2}}{2}} \beta^{2 n}}{\sqrt{2 n !}}
$$

and the normalization constant $N_{e}$ is given by

$$
\begin{aligned}
& N_{e}=\left[\sum _ { n = 0 } ^ { \infty } \frac { 1 } { 2 n ! } \left[|\alpha|^{4 n} e^{-|\alpha|^{2}}+|\beta|^{4 n}|\langle\beta \mid \alpha\rangle|^{2} e^{-|\beta|^{2}}\right.\right. \\
& \left.\left.-\alpha^{* 2 n} e^{-\frac{1}{2}\left(|\alpha|^{2}+|\beta|^{2}\right)}\langle\beta \mid \alpha\rangle \beta^{2 n}-\beta^{* 2 n} e^{\left.-\left.\frac{1}{2}|| \alpha\right|^{2}+|\beta|^{2}\right)}\langle\alpha \mid \beta\rangle \alpha^{2 n}\right]\right]^{1 / 2}
\end{aligned}
$$

Where

$$
|\langle\beta \mid \alpha\rangle|^{2}=e^{-|\beta-\alpha|^{2}}
$$




\section{2 (b) OSemi-CSs:}

From equation (13) OSemi-CSs is given as

$$
\left|\alpha_{\perp} \beta\right\rangle_{o}=\frac{1}{N_{o}} \sum_{n=0}^{\infty} C_{2 n+1}|2 n+1\rangle
$$

Where,

$$
C_{2 n+1}=\frac{1}{N_{o}} \frac{e^{-\frac{|\alpha|^{2}}{2}} \alpha^{2 n+1}-\langle\beta \mid \alpha\rangle e^{-\frac{|\beta|^{2}}{2}} \beta^{2 n+1}}{\sqrt{(2 n+1) !}}
$$

The normalization constant $N_{o}$ is also given by

$$
\begin{aligned}
& N_{o}=\left[\sum _ { n = 0 } ^ { \infty } \frac { 1 } { ( 2 n + 1 ) ! } \left[|\alpha|^{4 n+2} e^{-|\alpha|^{2}}+|\beta|^{4 n+2}|\langle\beta \mid \alpha\rangle|^{2} e^{-|\beta|^{2}}\right.\right. \\
& \left.\left.-\alpha^{* 2 n+1} e^{-\frac{1}{2}\left(|\alpha|^{2}+|\beta|^{2}\right)}\langle\beta \mid \alpha\rangle \beta^{2 n+1}-\beta^{* 2 n+1} e^{-\frac{1}{2}\left(|\alpha|^{2}+|\beta|^{2}\right)}\langle\alpha \mid \beta\rangle \alpha^{2 n+1}\right]\right]^{1 / 2}
\end{aligned}
$$

\section{Nonclassical Properties of Even and Odd Semi-CSs}

Nonclassical properties may be established through investigating quadrature squeezing, Sub-Poissonian distributions, The Husimi Q-function and phase distribution of the quantum states. In this section, we express these quantities with respect to even and odd semi-CSs which have been introduced in (14) and (17), respectively. Recall that a common feature of all of the mentioned criteria is that the Glauber-Sudarshan Pfunction [29] of the corresponding field state is not positive definite, i.e. it cannot be illustrated as a (classical) probability distribution. But, as a well-known fact, we would like to imply that finding this function is ordinarily a hard task. Altogether, each of the above signs, which will be considered in this paper, is indeed sufficient (not necessary) for a state to belong to nonclassical states. It should be mentioned that the necessary and sufficient criterion for the nonclassicality of a state is the subject of a recent research work [30].

\subsection{Quadrature Squeezing}

In order to study quadrature squeezing [31], we consider the following quadrature operators:

From which we have

$$
\hat{x}=\left(\hat{a}+\hat{a}^{+}\right) / 2 \text { and } \hat{p}=\left(\hat{a}-\hat{a}^{+}\right) / 2 i \text {. }
$$

$$
[\hat{x}, \hat{p}]=i \hat{C}, \quad \text { with } \quad \hat{C}=\frac{1}{2}
$$

Then the uncertainty relation holds $\left\langle(\Delta \hat{x})^{2}\right\rangle\left\langle(\Delta \hat{p})^{2}\right\rangle \geq \frac{1}{4}\langle\hat{C}\rangle^{2}$ where $\left\langle\left(\Delta \hat{x}_{i}\right)^{2}\right\rangle=\left\langle\hat{x}_{i}\right\rangle^{2}-\left\langle\hat{x}_{i}\right\rangle^{2}, \hat{x}_{i}=\hat{x}$ or $\hat{p}$ . A state is squeezed if any of the following conditions holds:

$$
\left\langle(\Delta \hat{x})^{2}\right\rangle<\frac{1}{4} \quad \text { or } \quad\left\langle(\Delta \hat{p})^{2}\right\rangle<\frac{1}{4} .
$$

Now by using Eqs. (20) and (21) the quadrature variances are given in terms of annihilation and creation operators expectation values by

$$
\begin{gathered}
\left\langle(\Delta \hat{x})^{2}\right\rangle=\frac{1}{4}+\frac{1}{2}\left[\operatorname{Re}\left\langle\hat{a}^{2}\right\rangle+\langle\hat{n}\rangle\right], \\
\left\langle(\Delta \hat{p})^{2}\right\rangle=\frac{1}{4}-\frac{1}{2}\left[\operatorname{Re}\left\langle\hat{a}^{2}\right\rangle-\langle\hat{n}\rangle\right]
\end{gathered}
$$

The state would possess $\hat{x}$ or $\hat{p}$ quadrature frequency sum squeezing if the $S$-factor defined by

$$
S_{X}=\frac{2\left\langle(\Delta \hat{x})^{2}\right\rangle-\langle\hat{C}\rangle}{\langle\hat{C}\rangle}, \quad \text { or } \quad S_{p}=\frac{2\left\langle(\Delta \hat{p})^{2}\right\rangle-\langle\hat{C}\rangle}{\langle\hat{C}\rangle}
$$


Satisfies the inequalities $-1 \leq S_{X}<0$ or $-1 \leq S_{p}<0$. For even or odd semi-CSs $\langle\hat{a}\rangle=\left\langle\hat{a}^{+}\right\rangle=0$. Also the expectation values of the square annihilation operator and the mean photon number for even and odd semiCSs are respectively given by

$$
\begin{aligned}
\left\langle\hat{a}^{2}\right\rangle_{e} & =\sum_{n=0}^{\infty}\left|C_{2 n}\right|^{2} \sqrt{2 n(2 n-1)}, \\
\langle\hat{n}\rangle_{e} & =\sum_{n=0}^{\infty}\left|C_{2 n}\right|^{2}(2 n), \\
\left\langle\hat{a}^{2}\right\rangle_{o} & =\sum_{n=0}^{\infty}\left|C_{2 n+1}\right|^{2} \sqrt{2 n(2 n+1)}, \\
\langle\hat{n}\rangle_{o} & =\sum_{n=0}^{\infty}\left|C_{2 n+1}\right|^{2}(2 n+1) .
\end{aligned}
$$

In Fig. 1, the squeezing function corresponding to states $\left|\alpha_{\perp} \beta\right\rangle_{e}$ and $\left|\alpha_{\perp} \beta\right\rangle_{o}$ versus the parameters $\alpha$ and $\beta$ have been plotted. These figures show that for both states, $S_{x}$ are always negative, and $S_{p}$ are always positive for all values of $\alpha$ and $\beta$, see Fig. 1 (a, c) and (b, d) respectively. This means that squeezing occurs, for both states, only in the x quadrature and so nonclassicality feature of these states is revealed.

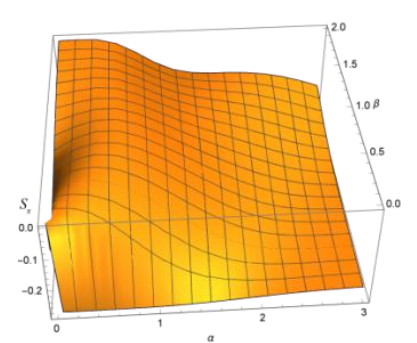

( a )

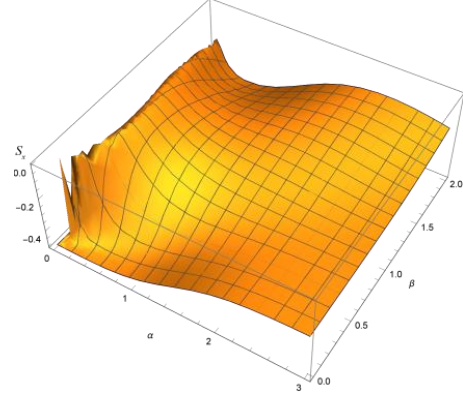

( c )

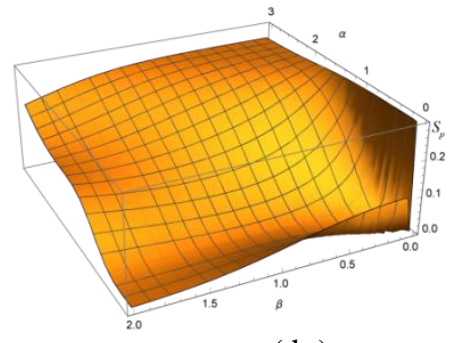

( b )

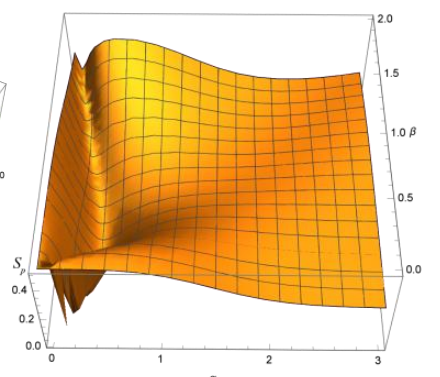

( d )

Fig. 1 The squeezing function $S_{x}$ and $S_{p}$ as a function of the parameters $\alpha$ and $\beta$ (a and b) for the even semiCSs and (c and d) for the odd semi-CSs.

\subsection{Sub-Poissonian Distributions}

In this subsection we shall investigate the sub-Poissonian distributions for the even and odd semi-CSs. The distribution can be measured by using photon detectors based on photoelectric effect [32;33]. The second-order correlation function, denoted $g^{2}(0)$, takes the following simple form [34],

$$
g^{2}(0)=\frac{\left\langle\hat{a}^{+2} a^{2}\right\rangle}{\left\langle a^{+} a\right\rangle^{2}}
$$

This expression is easily rewritten in terms of the photon number operator and can then be evaluated for even and odd semi-CSs using Eqs. (25,) and (27) as 


$$
g^{2}(0)=\frac{\left\langle\hat{n}^{2}\right\rangle-\langle\hat{n}\rangle}{\langle\hat{n}\rangle^{2}}
$$

If $g^{(2)}(0)<1(>1)$, then the light field exhibits sub(super)-Poissonian distribution; if $g^{(2)}(0)=1$ the distribution is called Poissonian; if $g^{(2)}(0)=2(>2)$ the distribution is referred to as thermal (super-thermal).

Fig. 2 (a, b) represents the second order correlation function for the even and odd semi-CSs respectively. As shown in this figure, for even semi-CSs $g^{2}(0)>2$ in a specific regions of $\alpha$ and $\beta$ indicating super-thermal-Poissonian distribution then with increasing both $\alpha$ and $\beta$ the function becomes super-Poissonian. In case of the odd semi-CSs, $g^{2}(0)>1$, in a specific regions of $\alpha$ and $\beta$, this means that it shows superPoissonian distribution. With increasing both $\alpha$ and $\beta$ parameters the function changes gradually to subPoissonian distribution. This means that both states has nonclassicality feature.

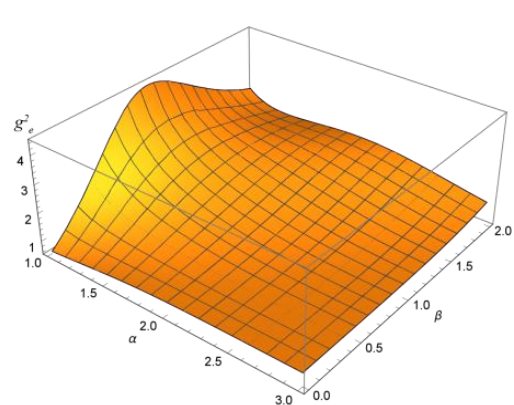

( a )

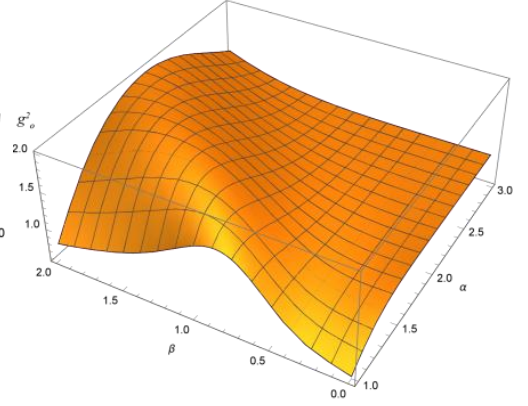

( b )

Fig. 2 Plot of the second order correlation function as a function of $\alpha$ and $\beta$, (a) for the even semi-CSs and (b) for the odd semi-CSs.

\subsection{The Husimi - Kano Q-function}

In this subsection we shall consider the Husimi - Kano Q-function associated with the even and odd semi-CSs. The Q-function is positive-definite at any point in the phase space for any quantum state and can be also calculated in terms of the following formula [35]

$$
Q(\gamma)=\pi^{-1}\langle\gamma|\rho| \gamma\rangle
$$

where $\rho$ is the density matrix for the even and odd semi-CSs and $|\gamma\rangle$ is the usual coherent state.[2] Thus the Qfunction for light fields in the even and odd semi-CSs will be given respectively, by

\section{3 (a) For the ESemi-CSs}

$$
Q_{e}(\gamma)=\pi^{-1}\left|G_{e}\right|^{2} e^{-|\gamma|^{2}}
$$

where

$$
G_{e}=\sum_{n=0}^{\infty} \frac{\gamma^{* 2 n}}{\sqrt{2 n !}} C_{2 n}
$$

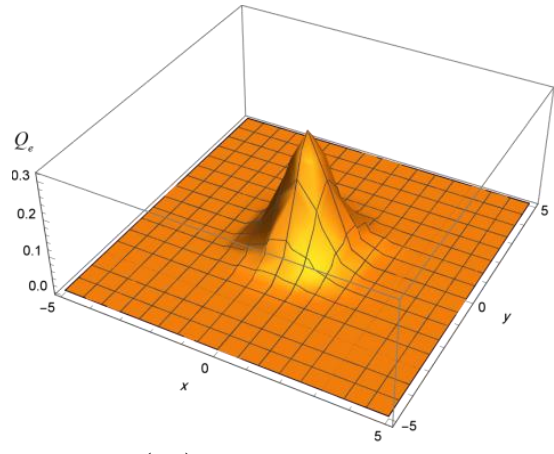

( a )

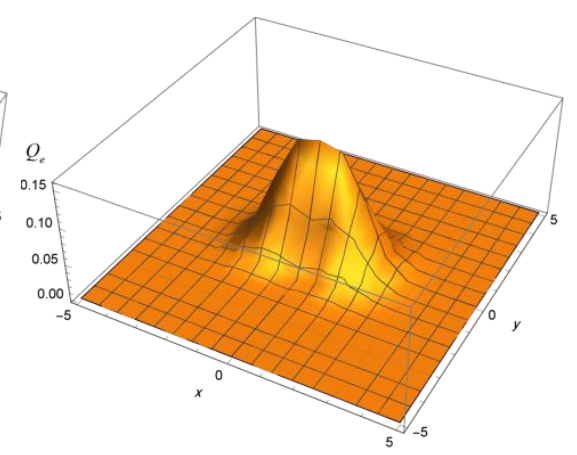

( b ) 


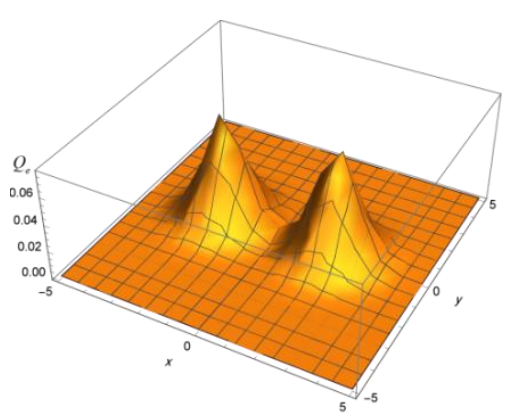

( c )

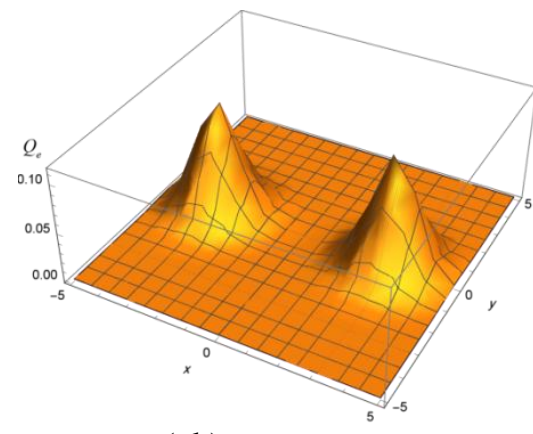

(d)

Fig. 3 The $Q_{e}$ - function against $\gamma$ for even semi-CSs at fixed $\beta=2$ (a) $\alpha=0$, (b) $\alpha=1$, (c) $\alpha=2$ and (d) $\alpha=3$.

Since the maximization or minimization of the $Q_{e}(\gamma)$-function depends on the parameter $\alpha$. Therefore, our main task is to examine the behaviour of the $Q_{e}(\gamma)$-function due to the variation in the $\alpha$ parameter. For this reason we plot $Q_{e}(\gamma)$ in Fig. (3) for different values of the $\alpha$-parameter keeping $\beta$ parameter unchanged. For fixed $\beta=2$ and for instance when we consider $\alpha=0$, the function exhibits one peak but with squeezing apparent on the contours of the base, see Fig.3 (a). For $\alpha=1$, we note that more squeezing occur on the contours of the peak base where it is elliptically shaped, see Fig.3 (b). For $\alpha=2$, we note that the peak splits into two peaks but they are joined near of the base and each base has a crescent like shape as shown in Fig.3 (c). More increase in the value of $\alpha=3$ leads to a complete separation of the two peaks, see Fig. 3 (d). It is also noted that there is a slight difference between the heights of the peaks. This means that there is a slight asymmetry in the function shape which reflects the effect of the squeezing.

\section{3 (b) For the OSemi-CSs}

$$
Q_{o}(\gamma)=\pi^{-1}\left|G_{o}\right|^{2} e^{-|\gamma|^{2}}
$$

where

$$
G_{o}=\sum_{n=0}^{\infty} \frac{\gamma^{* 2 n+1}}{\sqrt{(2 n+1) !}} C_{2 n+1}
$$

Where $C_{2 n}$ and $C_{2 n+1}$ are given by Eqs.(15) and (18), respectively.

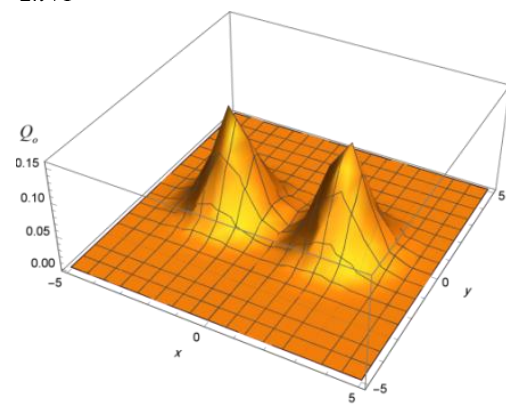

( a )

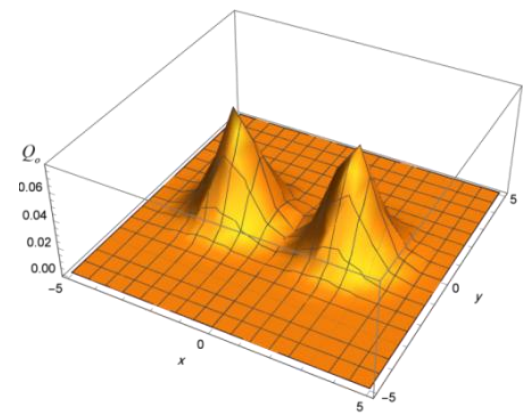

(c)

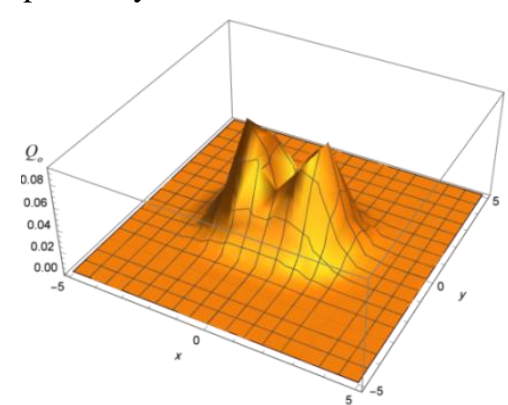

( b )

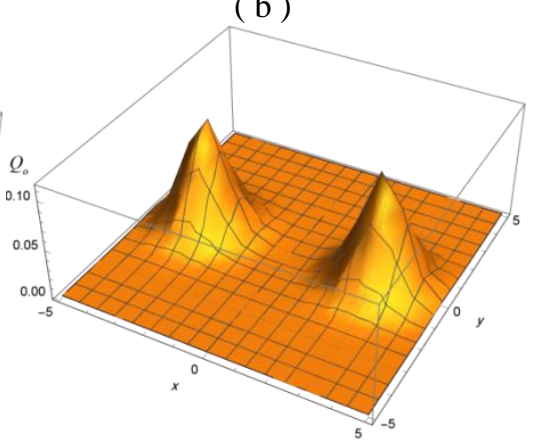

(d)

Fig. 4 The $Q_{o}(\gamma)$ - function against $\gamma$ for odd semi-CSs at fixed $\beta=2$ (a) $\alpha=0$, (b) $\alpha=1$,

(c) $\alpha=2$ and (d) $\alpha=3$. 
In order to visualize the behaviour of $Q_{o}(\gamma)$-function we plot the $Q_{o}(\gamma)$-function in Fig. (4) for the same values of the $\alpha$ and $\beta$-parameters as in $Q_{e}(\gamma)$. The function exhibits two peaks but with squeezing apparent on the contours of the base at $\alpha=0$, see Fig.4 (a). In Fig.4 (b), for $\alpha=1$, we note that the function shows a two-peak squeezed structure with pattern of interference at the base. From Fig. 4 (c), it is seen that, for $\alpha=2$, the function has the same behavior as in the case $\alpha=0$ with a decrease in the peak height. More increase in the value of $\alpha=3$ leads to a complete separation of the two peaks, see Fig. 4 (d). Generally, a slight difference between the heights of the peaks has been observed with changing $\alpha$ value. Also, the slight asymmetry in the function shape may be due to the effect of the squeezing.

\subsection{Phase distribution}

Phase distribution of a quantum state of the electromagnetic field provides information about uncertainty in the phase of the field. This, in turn, imposes a lower limit on the fluctuations in the number of photons. A useful approach to define phase distribution is the Pegg-Barnett formulation [36]. Phase distribution of an arbitrary state of the field is obtained by the overlap of the state with the phase state. Pegg and Barnett defined a complete set of $\mathrm{s}+1$ orthonormal phase states $\left|\theta_{p}\right\rangle$ by

$$
\left.\left|\theta_{p}\right\rangle=\frac{1}{\sqrt{s+1}} \sum_{n=0}^{s} \exp \left(\operatorname{in} \theta_{p}\right) n\right\rangle,
$$

Where $\theta_{p}=\theta_{0}+\frac{2 \pi p}{s+1}$ with $p=0,1, \ldots \ldots, s$ and an arbitrary value of $\theta_{0}$ based on these phase states, the Hermitian phase operator is defined as

$$
\hat{\phi}_{\theta}=\sum_{p=0}^{s} \theta_{p}\left|\theta_{p}\right\rangle\left\langle\theta_{p}\right|
$$

Henceforth, the Pegg-Barnett phase probability distribution of an arbitrary state $|\psi\rangle$ may be defined as

$$
p(\theta)=\lim _{s \rightarrow \infty} \frac{s+1}{2 \pi}\left|\left\langle\theta_{p} \mid \psi\right\rangle\right|^{2} .
$$

Now inserting the introduced states (14) and (17) into (34), the respective phase distributions for the states $\left|\alpha_{\perp} \beta\right\rangle_{e}$ and $\left|\alpha_{\perp} \beta\right\rangle_{o}$ are

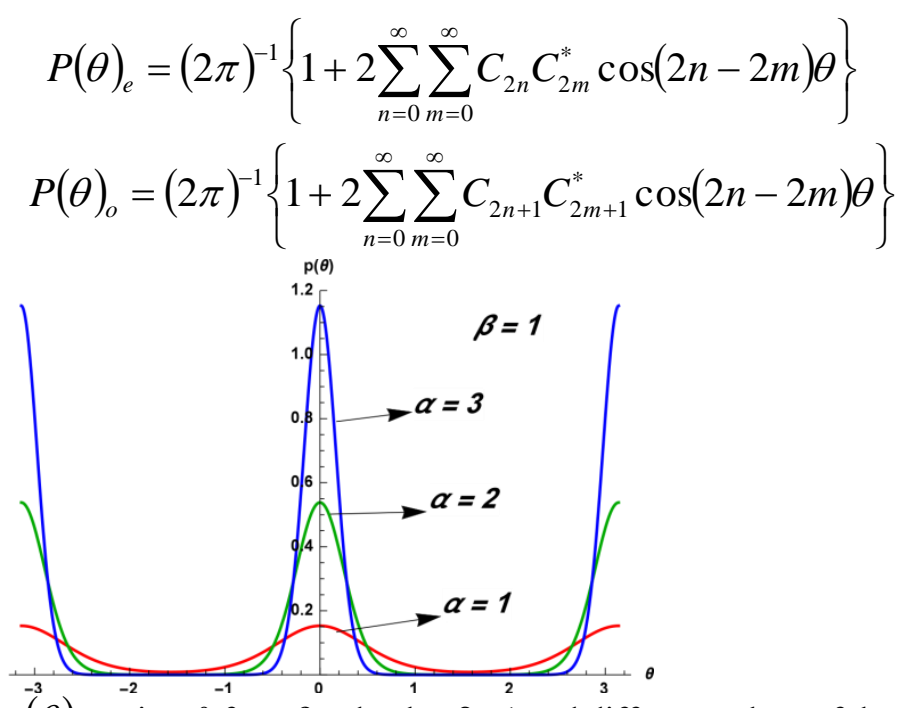

Fig. 5 The phase distribution $P_{P B}(\theta)$ against $\theta^{-1}$ for a fixed value $\beta^{0}=1$ and different values of the parameter $\alpha=$ 1,2 and 3 , for even semi-CSs.

In Fig. 5, we have plotted the Pegg-Barnett phase distribution for even semi-CSs for various values of $\alpha=1,2$ and 3 keeping $\beta$ fixed at 1 . Generally, for even semi-CSs, the figure show that all of the graphs have single peaks at $\theta=0$ and the distribution is symmetric around this peaks with two lateral wings at $\theta= \pm \pi$ 
developing. As $\alpha$ increase, the distribution $P_{P B}(\theta)$ peaks become sharper and higher. In fact, the same results and behavior have been seen also for odd semi-CSs.

\section{Conclusion}

In this article we have introduced and discussed some nonclassical properties of even and odd semicoherent states. The quadrature squeezing is investigated. It shows that both even and odd semi-CSs exhibit quadrature squeezing in $\mathrm{x}$ component. The second order correlation function for even and odd semi-CSs is studied. It shows that even and odd semi-CSs exhibits super-thermal-Poissonian and super-Poissonian distribution in a specific regions of $\alpha$ and $\beta$ respectively. On the other hand even and odd semi-CSs with increasing $\alpha$ and $\beta$ parameters changes to super and sub-Poissonian distribution respectively. The Husimi - Kano Q-function associated with the even and odd semi-CSs reveals that Q-function for both states are positivedefinite at any point in the phase space with a slight asymmetry in the function shape which reflects the effect of the squeezing. Also Q-function for both states tends to split up into two peaks as $\alpha$-parameter increases. Finally the phase properties using the Pegg-Barnett formalism are studied for even and odd semi-CSs. For both states the phase distribution function has one central peak at $\theta=0$ with two lateral wings at $\theta= \pm \pi$ developing and as $\alpha$ increase, the distribution $P_{P B}(\theta)$ peaks become sharper and higher.

\section{Acknowledgments}

The auther would like to thank Mrs. S. Sanad of the Mathemtics Depat-ment, Faculty of Science, Al-Azhar University (Girls Branch) for helpingin the programs for the figures.

\section{References}

[1] E. Schrodinger, Naturwissen schaften 14, (1926), 664.

[2] R. Glauber, Phys. Rev. 130, (1963), 2529.

[3] R. Glauber, Phys. Rev. 131, (1963), 2766.

[4] J.R. Klauder, J. Math. Phys. 4, (1963), 1055.

[5] J.R. Klauder, J. Math. Phys. 4, (1963), 1058

[6] H.J. Kimble, M. Dagenais and L. Mandel, Phys. Rev. Lett. 39, (1977), 691

[7] R. Short and L. Mandel, Phys. Rev. Lett. 51, (1983), 384.

[8] R.L.de Matos Filho and W.Vogel, Phys. Rev. Lett. 76, (1996), 608.

[9] V.I.Manko, G.Marmo, E.C.G.Sudarshan and F.Zaccaria, Phys. Scr. 55, (1997) 528.

[10] R.L.de Matos Filho and W.Vogel, Phys. Rev. Lett. A54, (1996), 4560.

[11] Z. Kis, W.Vogel and L.Davidovich, Phys. Rev. A 64, (2001), 033401

[12] G.S.Agarwal and K.Tara, Phys. Rev. A 43, (1991), 492.

[13] S.Sivakumar, J. Phys. A: Math. Gen. 32,(1999), 3441.

[14] A.Dehghani and B.Mojaveri, J. Phys. A: Math. Theor. 45, (2012), 095304.

[15] B.Mojaveri and A.Dehghani, Eur. Phys. J. D 67, (2013), 179.

[16] V.V.Dodonov, I.A.Malkin and V.I.Man'ko, Physica 72, (1974), 597.

[17] V.V.Dodonov and M.B.Reno, J. Phys. A: Math. Gen. 39, (2006) 7411.

[18] R.L.de Matos Filho and W.Vogel, Phys. Rev. A 58, (1998), 1661

S.Wallentowitz and W.Vogel, Phys. Rev. A 58, (1999), 679.

S.Wallentowitz and W.Vogel, Phys. Rev. A 59, (1999), 531.

[19] M.J. Faghihi and M.K.Tavassoly, Commun. Theor. Phys. 56, (2011), 327.

[20] E.M.F. Curado, J-P Gazeau and M.C.S Rodrigues Ligia, Phys. Scr. 82, (2010), 038108.

[21] J.B. Geloun and M.N. Hounkonnou, J. Phys. A: Math. Theor. 40, (2007), F817.

[22] M. Harouni Bagheri, R. Roknizadeh and M.H. Naderi, J. Phys. B: At. Mol. Opt. Phys. 41, (2008), 225501.

[23] P.M. Mathews and K. Eswaran, Nuovo Cimento B 17, (1973), 332-5.

[24] V.V. Dodonov, M.B. Horovits, , J. Phys. A: Math. Gen. 47, (2014), 315301

[25] A.Dehghani and B.Mojaveri, Int J. Theor.Phys. 54, (2015), 3507-3515.

[26] E. Piroozi and M.K.Tavassoly, J. Phys. A: Math. Theor. 45, (2012), 135301.

[27] L. Landau and E. Lifchitz, Quantum Mechanics: Non-Relativistic Theory, (Pergamon, New York, (1977).

[28] A.Dehghani, H. Fakhri, B. Mojaveri, J. Math. Phys. 53, (2012), 123527.

[29] E.C.J. Sudarshan, Phys. Raev. Lett. 10, (1963), 277.

[30] E.V. Shchukin and W. Vogel, Phys. Rev. A 72, (2005), 043808.

[31] D.F. Walls, Nature 306, (1983), $14 \mathrm{a} 1$.

[32] A. S. F. Obada and E. M. Khalil, Opt. Commun. 260, (2006), 19.

[33] E. M. Khalil, J. Phys. A: Math. Gen. 39, (2006), 11053.

[34] J. S. Wang and X. G. Meng, Chin. Phys. 16, (2007), 2422.

[35] A. S. F. Obada and M. Darwish, J. Mod. Opt. 51, (2004), 209.

[36] D.T.Pegg and S.M. Barnett, (1989), Phys. Rev. A 39, 1665-1675. 